\title{
Similarity-Based Clustering for Enhancing Image Classification Architectures
}

\author{
Dishant Parikh \\ Computer Engineering \\ GCET \\ Gujarat, India \\ dishant30899@gmail.com
}

\author{
Shambhavi Aggarwal \\ Information Technology \\ GCET \\ Gujarat, India \\ agg.shambhavi@gmail.com
}

\begin{abstract}
Convolutional networks are at the center of best in class computer vision applications for a wide assortment of undertakings. Since 2014, profound amount of work began to make better convolutional architectures, yielding generous additions in different benchmarks. Albeit expanded model size and computational cost will, in general, mean prompt quality increases for most undertakings but, the architectures now need to have some additional information to increase the performance. We show empirical evidence that with the amalgamation of content-based image similarity and deep learning models, we can provide the flow of information which can be used in making clustered learning possible. We show how parallel training of sub-dataset clusters not only reduces the cost of computation but also increases the benchmark accuracies by 5-11 percent.

Index Terms-Image similarity, Image Classification, CNN, Dataset Class Similarity Index
\end{abstract}

\section{INTRODUCTION}

Understanding the world in a solitary look is one of the most practiced accomplishments of the human mind. It takes only milliseconds to perceive the classification of an object or an action, underlining a significant job of feedforward processing in visual analysis. With the introduction of deep convolutional neural networks [1], which achieved breakthrough accuracies in the domain of image classifications, there has been a profuse amount of work in developing better architectures and training methodologies [2] [3]. The current work, in image classification, is more focused on the networks and lesser when it comes to the understanding of the dataset and its structure. For instance, we saw the work on depthwise separable network architectures [4] [5] and dynamic model scaling [6], with the methodologies like the Noisy student's semi-supervised learning [7], which brought a higher accuracy on ImageNet while providing an intuition into self-learning and distillation. Although structures like these have produced astonishing results on many of the large databases like ImageNet [8], they still need to be tweaked and trained in a particular way to get the best of object detection [9], while dealing with finegrained datasets [10].

We have seen a significant improvement in accuracy by tuning the models based on knowledge acquired from studying the structure of the dataset. So what if we append that type of information to the model itself?

The answer came from a field that has yet not been amal- gamated, with modern deep learning architectures: Image similarity [11]. Since the foundation of the field, we saw a ton of work in understanding the manner in which machines see an image. For example, when image fusion evolved with the idea to make the output image show the understanding of the scene more elaborately than all the input images before, it was a sincere thought into the development of image retrieval systems [12] [13] [14] [15]. And by combining the use of deep learning to understand the content-based image similarities, it was now easier to develop accurate analysis models.

We show how by amalgamating these two fields, we achieve even better architectures. We show the need for an additional meta-data field, which we call: Similarity Index. It could help save hours of computational time and also increase the accuracy of the deepest of the architectures on class-similar datasets. While working with the datasets which contain a hodgepodge of similar classes and distinctive ones, for example, Stanford's dog breeds dataset [10], it is difficult to tune the model efficiently for better performance. The problem is not only the drop in accuracy but also the overfitting of the model. The standard techniques could only provide a way out of overfitting upto a certain limit [16].

\section{A. Clustered training}

What we propose is a clustering of the dataset into two or more sub-datasets, with parallel training to gain better accuracy. The clusters are formed based on a similarity matrix of all the classes inside the dataset, and hence we find the most similar classes together in one cluster. Once we generate the sub-dataset clusters, it is all too easy; we train independent models on each sub-dataset. The part of testing, when a new image is to be predicted, we check that against all the cluster's similarity indexes, and we pass it to the model it belongs to. With the use of feature extractions and distance calculations in the feature space of the two images, we successfully find the best model to go for making the prediction. This way, we reduce the computational needs as well as increase the model accuracies, even with a limited number of epochs.

\section{B. Similarity Index}

Additionally, we propose the idea of a similarity index, which could lead to a better selection of model architectures. 
The similarity index represents the overall dataset's class similarity. What is the use of this? The thing is that sometimes machines and humans have a different sense of what is difficult to distinguish. Sometimes, as developers, we might think that a particular dataset is too difficult to distinguish, and hence we use deeper architectures for better feature extraction. But maybe, the machine does not perceive them as difficult. What if we could already have a parameter, which could tell us when to use which type of architectures? It is when the Dataset Class Similarity Index (DCSI) comes to play. If we could append an overall class similarity index to the dataset under concern, then it can help anyone using the dataset to select better architecture for classification.

\section{RELATED WORKS}

Until recently, most of the work in Image classification has been done, by making the models deeper and the labeled databases larger. The recent work, in dynamic model scaling, did show the intent of tweaking the model by the hyperparameters, which can make it efficient without making it unnecessarily complicated [17]. The researchers have also shown much work in the model architecture's efficiency, like introducing inverted residuals and liner bottlenecks for lighter models [5] and descriptor pyramids [18].

Some work in the latest architecture revolved around the kernel functions [19]. We also saw the work in Deep sparse rectifier networks [20] and even in understanding the difficulties of training deeper networks [21]. We even saw some changes in base architectures to further improve performance [22], but mostly we thought that the deeper architectures and faster computational machinery would provide the accuracies that we want. Even though possible, sometimes, the problem of overfitting and the cost of computing becomes a huge hurdle. Even with state-of-the-art models, we are not able to achieve impressive accuracy when it comes to class-similar datasets. What we tried is to check the possibility of improving the methodology by amalgamating the knowledge, driven by image similarity of classes in the datasets.

Much work has been done, in understanding the image, by using content-based analysis [23] or even complex wavelet structural analysis [24]. But the work was mostly transferred to image retrieval systems. We saw work on using these analysis for making the application better [25], which was used not only on content-based image analysis but also objectbased image analysis [26]. Further, we saw the development of similarity engines [27] and the best-in-class content-based image retrievals. With the use of fundamentals of contentbased image analysis [14], we produce a similarity matrix for the entire dataset. The similarity between the classes does provide a critical insight into training better models. Not improving the architecture, but by improving how these architectures are trained.

We hence developed a generic methodology of clustering the data into sub-datasets and then train the models independently on them. Once we get the set of models, the new query image needs to make a correct choice of model to send to for prediction. We provide the clustered-model training method for faster training and more accurate models for class-similar datasets.

\section{Methodology}

\section{A. Content-based Similarity}

The first step into the development of the methodology was to find and build an accurate feature extraction and matching model, to have precise clustering of classes. We studied many possible options, but the best-in-class methodology was to use deep learning. We used the base methodology of a contentbased image retrieval (CBIR) system [12] for feature space development.

We used the ResNet152 model for performing feature extraction on all the images. The feature space hence contains all the features of images based on their class. The same feature space acts as an input for Algorithm 1.

\section{B. Datasets information}

For our experimentations, we used the Stanford dog breeds dataset [10] for checking out the methodology. The dataset contains 120 classes of different images, one for each breed. The reason for selecting this dataset is that it contained the appropriate mixture of image classes. Some breeds are too similar to classify properly with simple architectures, while some are too distinct and hence easier to classify. We thought it would be appropriate to run the proposed methodology on a dataset like this. The dataset contains over 20000 images, with about 150 samples for each of 120 classes. For further confirming the thesis, we used other datasets like the Stanford's Cars [28] and Oxford's Flower dataset [29].

\section{Class-similarity matrix}

Once the feature map was ready, we compared those features and calculated the similarity, as mentioned in Algorithm 1. The class-to-class similarity was calculated by checking the distance of the images in the first class, with the images in the second class. We took the average of the individual distances to label it as the overall closeness between two classes. For better efficiency, we checked the following distance metrics [30] [31]:

1) Cosine distance

2) Manhattan distance

3) L1 and L2 distances

4) Squared distance

We found that the distance, most accurate to our architecture, was the cosine distance. So, by selecting that, we calculated the similarity between all the image classes. What we got was a $n \times n$ matrix with similarity values, where $n$ represents the number of classes in the dataset.

\section{Class Clusters}

Once the similarity matrix was ready, which is the output of Algorithm 1, we needed an appropriate algorithm to cluster those classes, based solely on the similarity matrix. We had two options to view it through: the hierarchical clustering [32] 


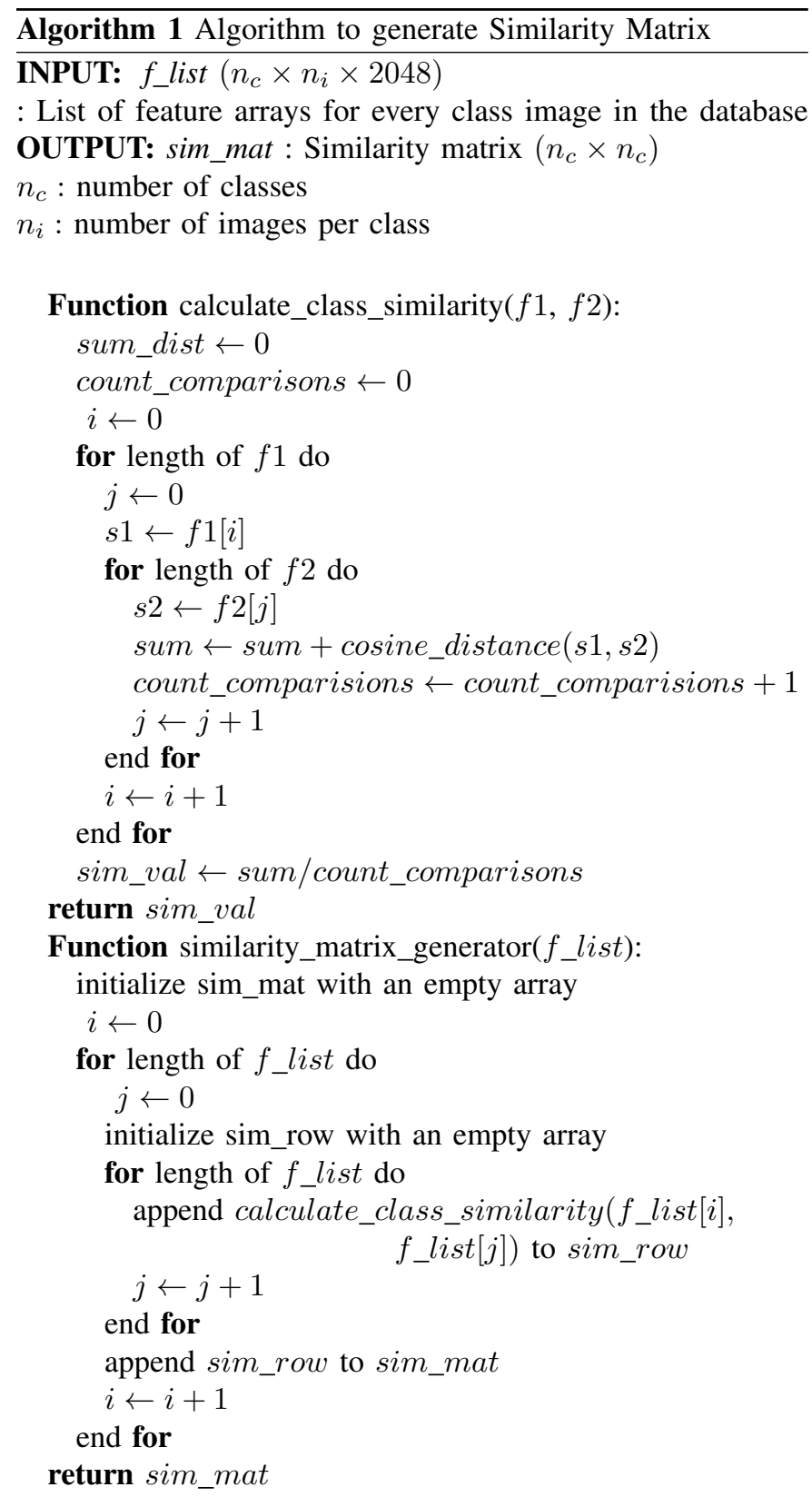

and the advanced k-means algorithm [33] [34]. We went with hierarchical clustering due to the possibility of governing the clustering decision by setting the linkage attribute. We used different linkages [35] based on the dataset requirement, and it is easy to test the performances likewise.

\section{E. Model architectures}

We wanted to use some of the state-of-the-art architectures and also the methodologies fine-tuned for image classification on the datasets under concern. The architectures we deployed for the base results, are as follows:

1) Pre-trained MobileNetv2 (ImageNet) + $\mathrm{GAP}+\mathrm{BN}+$ $\mathrm{FC}(1280)+\mathrm{BN}+$ SoftMax classifier

2) Pre-trained DenseNet121 (ImageNet) + GAP + BN + $\mathrm{FC}(1280)+\mathrm{BN}+$ SoftMax classifier $\overline{\text { Algorithm } 2 \text { Algorithm to choose the appropriate sub-model }}$ for predicting the new image

INPUT: cluster_hi_features $\left(n_{c} \times 2048\right)$ : Feature arrays for every sub-dataset cluster,

$\mathrm{i} €\{1, \ldots \mathrm{n}\}$, where $n$ is number of clusters

query_features: Feature array of the query image $(2048 \times 1)$

$n_{c}$ : number of images per class in the particular sub-dataset cluster

OUTPUT: Appropriate sub-dataset cluster model call

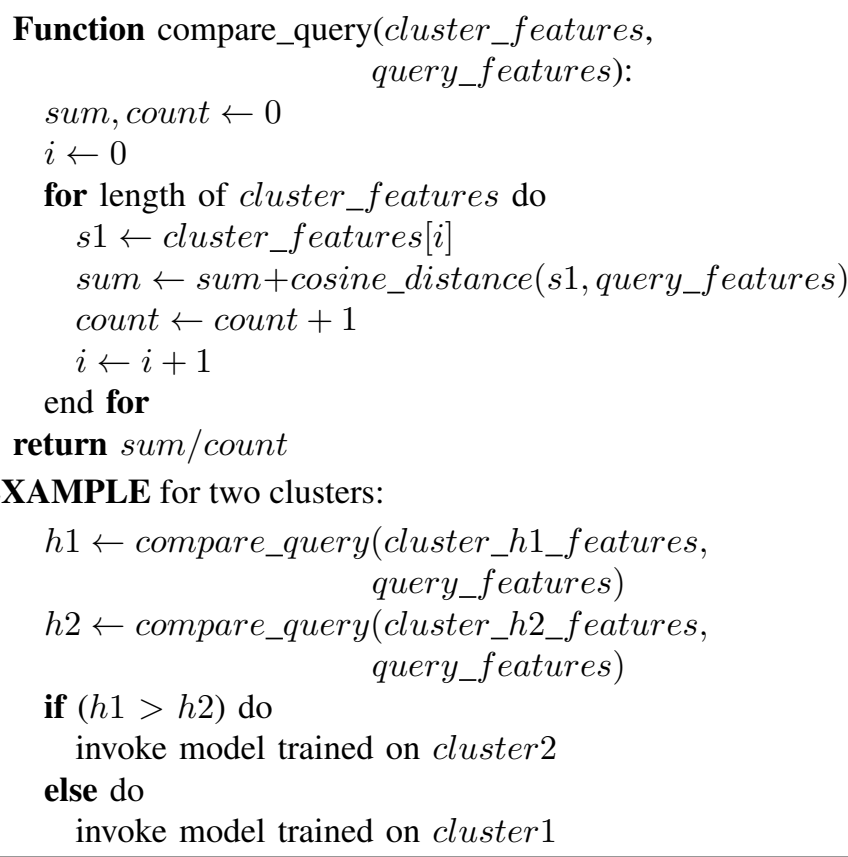

3) Pre-trained EfficientNetB7 (ImageNet) + GAP + SoftMax classifier

4) Pre-trained EfficientNetB7 (Noisy Student) + GAP + SoftMax classifier

5) Pre-trained InceptionResNetv2 (ImageNet) + GAP + SoftMax classifier

6) Pre-trained ResNet50 (ImageNet) + GAP + SoftMax classifier

7) Pre-trained ResNet50 (ImageNet) + GAP + FC(128) + $\mathrm{FC}(64)+$ SoftMax classifier (model will be referred to as: $\operatorname{ResNet}+\mathbf{C})$

Callbacks: ReduceLROnPlateau, EarlyStopping, LambdaCallback, Tensorboard, ModelCheckpoint

** GAP - Global Average Pooling, BN - Batch Normalization [14], FC - Fully Connected Layer, and the parenthesis following the model name shows the weights used.

We initialized the weights of dense layers using the Xavier uniform initializer and used ReLU activations. The SoftMax layer had randomly initialized weights. All layers were trainable, and we compiled the models using categorical crossentropy loss and Adam optimizer.

The criteria for training was simple. We wanted to keep limited iterations just as to stop the models from overfitting. We 
Fig. 1. Process Methodology

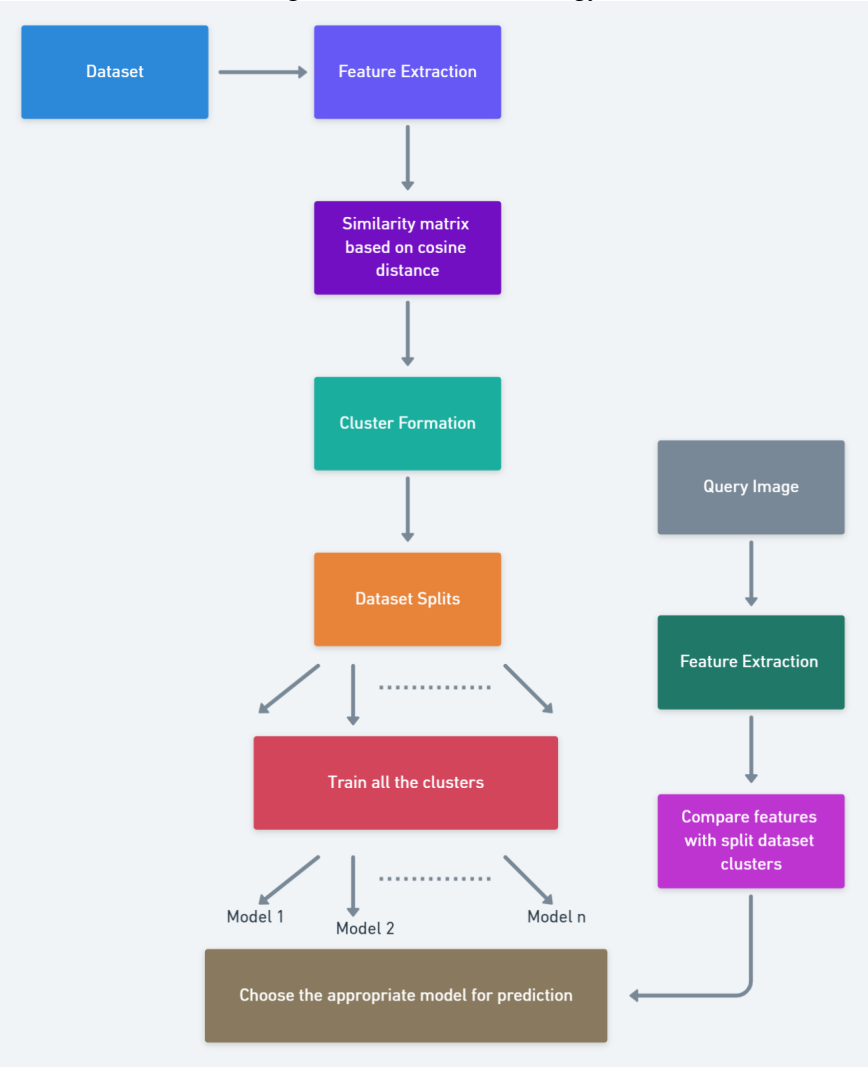

used the epochs as the stopping criteria and not the time of computations, although the same has been discussed in Section: Experiments and Discussions. We made sure that we have enough blend of architectures as we required to test the methodology on varying structures to improve the POC for scalability.

\section{F. Improved model architectures}

After the base model architecture definitions, it was time for making those efficient by invoking clustered training. The flow was simple. Decide the number of clusters needed and invoke the hierarchical clustering for that many clusters. Once individual cluster classes are defined, we train the same models on these sub-dataset clusters. But that was the simple part, the main thing to do was when the test image comes, we needed to match an appropriate model with it. It seemed like a tricky part but was not. We just required to extract the features of that image, and then compare it with the features of the clustered class images, as shown in Algorithm 2. What this means is that it needs to take one sample from each class of a given cluster, compare it with the query image, and then take an average as its similarity. So, once we get the similarity of each cluster with the query image, we just required to choose the class which had the closest similarity. Once we know which class it belongs to, we make the prediction based on the model of that particular cluster.

\section{EXPERIMENTS AND DISCUSSIONS}

As mentioned, we trained our architectures on varied datasets, including Stanford's dog breeds dataset, cars dataset [28] as well as Oxford's flower's dataset [29]. We trained it as per the criteria mentioned. As you can observe from Table I, there is an average performance by the base model architectures, on the Dog breeds dataset. We trained the models

TABLE I

ACCURACY OF THE MODEL ARCHITECTURES, TRAINED ON THE WHOLE DATASET.

\begin{tabular}{|c|c|}
\hline Architectures & Validation Accuracy \\
\hline MobileNetV2 & 78.44 \\
\hline DenseNet121 & 81.76 \\
\hline EfficientNetB7 & 90.62 \\
\hline Noisy-student & 88.542 \\
\hline InceptionResNetV2 & 86.46 \\
\hline ResNet50 & 87.5 \\
\hline ResNet50 + C & 80.208 \\
\hline
\end{tabular}

for 40 epochs each. Table I represents the validation accuracy while training the same model architectures on clusters formed by splitting the dataset into two, based on similarity. Table II represents the validation accuracy while training the same model architectures on clusters formed by splitting the dataset into two, based on similarity.

As you can see in Table II, there is a significant improvement in the model accuracies. Not only the average of, but even the accuracy on individual cluster(s), shows significant improvement. Now, this was just for two clusters. It is also important to determine a fitting value for the number of splits to be performed. Table III shows the accuracy for three cluster splits.

As you can see in that table, with a proper number of splits, the accuracies boost-up pretty well. And this can also lead to developing better models depending on how many clusters work well with that particular architecture. To confirm the thesis, we then tested the error rate of choosing the model for a new query image. The model choice prediction was tested on 10,000 query images. We got an overall error of $\mathbf{0 . 0 6 \%}$. That is quite negligible.

As seen in Figure 2, when we split the dataset based on the similarity, it increases the accuracy of the same architectures trained on entire dataset. It shows that a more focused dataset does make the model efficient. Figure 2 also supports the hypothesis, as we see that the accuracy graph of clusters is above the baseline architectures' accuracy.

What we see is that even after considering the error rate of choice, the model accuracies are better than the base model methodology. What we recommend is an added attribute in meta-data of the dataset: the similarity matrix. If we have a class similarity matrix then anyone who wants to use the matrix for clustering the dataset beforehand and then run the classifier, can do so, easily.

The experiments and the results stay true for other datasets 
TABLE II

ACCURACY OF THE MODEL ARCHITECTURES, TRAINED ON TWO SUB-DATASET CLUSTERS.

\begin{tabular}{|c|c|c|c|}
\hline Architectures & Validation Accuracy Cluster 1 & Validation Accuracy Cluster 2 & Validation Accuracy Cluster Avg. \\
\hline MobileNetV2 & 76.493 & 81.783 & 79.138 \\
\hline DenseNet121 & 83.955 & 86.822 & 85.3885 \\
\hline EfficientNetB7 & 96.875 & 84.375 & 90.625 \\
\hline Noisy-student & 96.875 & 87.5 & 92.1875 \\
\hline InceptionResNetV2 & 87.5 & 85.938 & 86.719 \\
\hline ResNet50 & 96.875 & 81.25 & 89.0625 \\
\hline ResNet50 + C & 93.75 & 79.68 & 84.715 \\
\hline
\end{tabular}

TABLE III

ACCURACY OF THE MODEL ARCHITECTURES, TRAINED ON THREE SUB-DATASET CLUSTERS.

\begin{tabular}{|c|c|c|c|c|}
\hline Architectures & Validation Accuracy Cluster 1 & Validation Accuracy Cluster 2 & Validation Accuracy Cluster 3 & Validation Accuracy Cluster Avg. \\
\hline MobileNetV2 & 75.74 & 82.249 & 82.709 \\
\hline DenseNet121 & 83.582 & 87.574 & 85.879 \\
\hline EfficientNetB7 & 96.875 & 93.75 & 95 \\
\hline Noisy-student & 93.75 & 93.75 & 85 \\
\hline InceptionResNetV2 & 96.875 & 100 & 90.67 \\
\hline ResNet50 & 93.75 & 100 & 95.2 & 95 \\
\hline ResNet50 + C & 93.75 & 100 & 95.63 & 925 \\
\hline
\end{tabular}

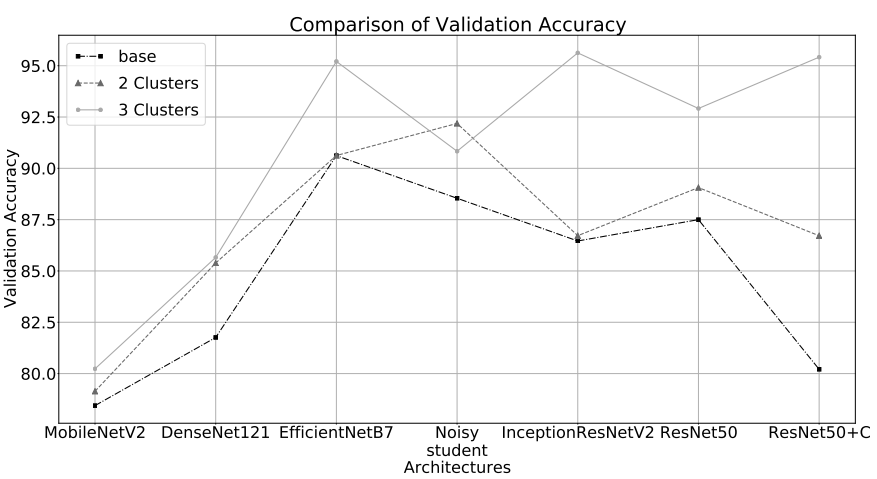

Fig. 2. Validation accuracy of architectures with and without clustering.

too. The varying number of clusters does eventually show a tweak in accuracy. So, the methodology can be adaptive with any kind of data but significantly for the datasets with mixed class similarity. And hence, if at the time when a dataset is released, we can add this parameter of similarity index, it can be helpful for the users to select an appropriate model. The similarity index will eliminate the trial and error phase of model selection that goes on in most cases.

\section{FUTURE WORKS AND CONCLUSION}

As we have seen that the methodology is adaptive, but still, more work can be done in improving the dynamic feature extractions as well as in reducing the error of choice. We can also work in formalizing the way Dataset class similarity index (DCSI) is defined. The clustering algorithms can be changed and chosen according to the way data is represented. Here we do leave the room for additional parameters on which the methodology can be improved, and comparative analysis with different clustering, number of clusters, and base model architectures can be performed. There is still possibility of improving the way the clusters are formed or the way the similarity is extracted. The methodology, however, has to be considered. We wanted to drive the deep learning architectures and the modern datasets into the choice of declaring the similarity index, to have better knowledge as well as decision capacity of choosing the correct model for classification.

We showed how the knowledge of similarity of the image classes, can be leveraged in making the model trainings better and faster. It can also be used when the computational capacity is low for a single thread. The dataset can be broken down easily into clusters, which are trained individually, and then a rule-based system can be provided to make the appropriate model choice for predictions.

\section{REFERENCES}

[1] Huang, G., Liu, Z., Van Der Maaten, L., \& Weinberger, K. Q. (2017). Densely connected convolutional networks. In Proceedings of the IEEE conference on computer vision and pattern recognition (pp. 4700-4708).

[2] Szegedy, C., Ioffe, S., Vanhoucke, V., \& Alemi, A. A. (2017, February). Inception-v4, inception-resnet and the impact of residual connections on learning. In Thirty-first AAAI conference on artificial intelligence.

[3] He, K., Zhang, X., Ren, S., \& Sun, J. (2016). Deep residual learning for image recognition. In Proceedings of the IEEE conference on computer vision and pattern recognition (pp. 770-778).

[4] Howard, A. G., Zhu, M., Chen, B., Kalenichenko, D., Wang, W., Weyand, T., ... \& Adam, H. (2017). Mobilenets: Efficient convolutional neural networks for mobile vision applications. arXiv preprint arXiv:1704.04861.

[5] Sandler, M., Howard, A., Zhu, M., Zhmoginov, A., \& Chen, L. C. (2018). Mobilenetv2: Inverted residuals and linear bottlenecks. In Proceedings of the IEEE conference on computer vision and pattern recognition (pp. 45104520).

[6] Tan, M., \& Le, Q. V. (2019). Efficientnet: Rethinking model scaling for convolutional neural networks. arXiv preprint arXiv:1905.11946. 
[7] Xie, Q., Luong, M. T., Hovy, E., \& Le, Q. V. (2020). Self-training with noisy student improves imagenet classification. In Proceedings of the IEEE/CVF Conference on Computer Vision and Pattern Recognition (pp. 10687-10698).

[8] Krizhevsky, A., Sutskever, I., \& Hinton, G. E. (2012). Imagenet classification with deep convolutional neural networks. In Advances in neural information processing systems (pp. 1097-1105).

[9] Bianco, S., Cadene, R., Celona, L., \& Napoletano, P. (2018). Benchmark analysis of representative deep neural network architectures. IEEE Access, 6, 64270-64277.

[10] Khosla, A., Jayadevaprakash, N., Yao, B., \& Li, F. F. (2011, June). Novel dataset for fine-grained image categorization: Stanford dogs. In Proc. CVPR Workshop on Fine-Grained Visual Categorization (FGVC) (Vol. 2, No. 1).

[11] Wang, J., Song, Y., Leung, T., Rosenberg, C., Wang, J., Philbin, J., ... \& Wu, Y. (2014). Learning fine-grained image similarity with deep ranking. In Proceedings of the IEEE Conference on Computer Vision and Pattern Recognition (pp. 1386-1393).

[12] Ciocca, G., \& Schettini, R. (2001). Content-based similarity retrieval of trademarks using relevance feedback. Pattern Recognition, 34(8), 16391655.

[13] Liu, Y., Zhang, D., Lu, G., \& Ma, W. Y. (2007). A survey of contentbased image retrieval with high-level semantics. Pattern recognition, 40(1), 262-282.

[14] Long, F., Zhang, H., \& Feng, D. D. (2003). Fundamentals of contentbased image retrieval. In Multimedia information retrieval and management (pp. 1-26). Springer, Berlin, Heidelberg.

[15] Wan, J., Wang, D., Hoi, S. C. H., Wu, P., Zhu, J., Zhang, Y., \& Li, J. (2014, November). Deep learning for content-based image retrieval: A comprehensive study. In Proceedings of the 22nd ACM international conference on Multimedia (pp. 157-166).

[16] Srivastava, Nitish and Hinton, Geoffrey and Krizhevsky, Alex and Sutskever, Ilya and Salakhutdinov, Ruslan. Dropout: a simple way to prevent neural networks from overfitting. The journal of machine learning research 15 (1) (2014) 1929-1958.

[17] Lu, Dengsheng and Weng, Qihao. A survey of image classification methods and techniques for improving classification performance. International journal of Remote sensing 28 (5) (2007) 823-870.

[18] Iandola, F., Moskewicz, M., Karayev, S., Girshick, R., Darrell, T., \& Keutzer, K. (2014). Densenet: Implementing efficient convnet descriptor pyramids. arXiv preprint arXiv:1404.1869.

[19] Liu, J. E., \& An, F. P. (2020). Image Classification Algorithm Based on Deep Learning-Kernel Function. Scientific Programming, 2020.

[20] Glorot, X., Bordes, A., \& Bengio, Y. (2011, June). Deep sparse rectifier neural networks. In Proceedings of the fourteenth international conference on artificial intelligence and statistics (pp. 315-323).

[21] Glorot, X., \& Bengio, Y. (2010, March). Understanding the difficulty of training deep feedforward neural networks. In Proceedings of the thirteenth international conference on artificial intelligence and statistics (pp. 249256).

[22] Szegedy, Christian and Vanhoucke, Vincent and Ioffe, Sergey and Shlens, Jon and Wojna, Zbigniew. Rethinking the inception architecture for computer vision. Proceedings of the IEEE conference on computer vision and pattern recognition, 2016, (pp. 2818-2826).

[23] Smeulders, A. W., Worring, M., Santini, S., Gupta, A., \& Jain, R. (2000). Content-based image retrieval at the end of the early years. IEEE Transactions on pattern analysis and machine intelligence, 22(12), 13491380 .

[24] Sampat, M. P., Wang, Z., Gupta, S., Bovik, A. C., \& Markey, M. K. (2009). Complex wavelet structural similarity: A new image similarity index. IEEE transactions on image processing, 18(11), 2385-2401.

[25] Agarwal, Ritika and Shankhadhar, Ankit and Sagar, Raj Kumar. Detection of lung cancer using content based medical image retrieval. 2015 Fifth International Conference on Advanced Computing \& Communication Technologies, IEEE, 2015, (pp. 48-52).

[26] Hoiem, Derek and Sukthankar, Rahul and Schneiderman, Henry and Huston, Larry. Object-based image retrieval using the statistical structure of images. Proceedings of the 2004 IEEE Computer Society Conference on Computer Vision and Pattern Recognition, 2004. CVPR 2004, Vol. 2, IEEE, 2004, (pp. II-II).

[27] Jain, R., Horowitz, B., Fuller, C. E., Gupta, A., Bach, J. R., \& Shu, C. F. (1999). U.S. Patent No. 5,893,095. Washington, DC: U.S. Patent and Trademark Office.
[28] Krause, J., Stark, M., Deng, J., \& Fei-Fei, L. (2013). 3D Object Representations for Fine-Grained Categorization. 2013 IEEE International Conference on Computer Vision Workshops. doi:10.1109/iccvw.2013.77

[29] Nilsback, M. E., \& Zisserman, A. (2008, December). Automated flower classification over a large number of classes. In 2008 Sixth Indian Conference on Computer Vision, Graphics \& Image Processing (pp. 722729). IEEE.

[30] Bar-Hillel, A., Hertz, T., Shental, N., \& Weinshall, D. (2003). Learning distance functions using equivalence relations. In Proceedings of the 20th International Conference on Machine Learning (ICML-03) (pp. 11-18).

[31] Hasnat, A., Halder, S., Bhattacharjee, D., Nasipuri, M., \& Basu, D. K. (2013). Comparative study of distance metrics for finding skin color similarity of two color facial images. ACER: New Taipei City, Taiwan, 99-108

[32] Vijaya, A. S., \& Bateja, R. (2017). A Review on Hierarchical Clustering Algorithms. J. Eng. Appl. Sci, 12(24), 7501-7507.

[33] Kanungo, T., Mount, D. M., Netanyahu, N. S., Piatko, C. D., Silverman, R., \& Wu, A. Y. (2002). An efficient k-means clustering algorithm: Analysis and implementation. IEEE transactions on pattern analysis and machine intelligence, 24(7), 881-892.

[34] Baruri, R., Ghosh, A., Banerjee, R., Das, A., Mandal, A., \& Halder, T. (2019, February). An Empirical Evaluation of k-Means Clustering Technique and Comparison. In 2019 International Conference on Machine Learning, Big Data, Cloud and Parallel Computing (COMITCon) (pp. 470475). IEEE.

[35] Sharma, S., \& Batra, N. (2019, February). Comparative Study of Single Linkage, Complete Linkage, and Ward Method of Agglomerative Clustering. In 2019 International Conference on Machine Learning, Big Data, Cloud and Parallel Computing (COMITCon) (pp. 568-573). IEEE.

[36] Ioffe, S., \& Szegedy, C. (2015). Batch normalization: Accelerating deep network training by reducing internal covariate shift. arXiv preprint arXiv:1502.03167. 